\title{
La eliminación del sarampión en Cuba
}

\author{
Miguel A. Galindo, ${ }^{1}$ Manuel Santín, ${ }^{1}$ Sonia Resik, ${ }^{2}$ María \\ de los Ángeles Ribas, ${ }^{2}$ María Guzmán,, ${ }^{2}$ Pedro Mas Lago, ${ }^{2}$ \\ Marc Strassburg, ${ }^{3}$ Bradley S. Hersh ${ }^{4}$ y Ciro A. de Quadros ${ }^{4}$
}

RESUMEN La vacuna antisarampionosa se empezó a usar en Cuba en 1971. En los años setenta se implantó una estrategia inicial para el control del sarampión, y a ella le siguieron iniciativas adicionales a principios de los años ochenta. Pese a haberse mejorado el programa de control, siguieron produciéndose brotes de la enfermedad. En 1986, después de revisar las experiencias recogidas a partir de las iniciativas de control ya establecidas, se adoptó una nueva estrategia de vacunación antisarampionosa.

Con el tiempo, la nueva estrategia de vacunación contra el sarampión llegó a tener tres componentes principales: primero, una campaña única de vacunación de "puesta al día" dirigida a niños de 1 a 14 años de edad. Segundo, se procuró lograr y mantener una alta cobertura con la vacuna mediante el ofrecimiento de servicios de vacunación obligatoria para niños de 12 meses de edad ("vacunación de mantenimiento"). Por último, se llevaron a cabo periódicamente campañas de "seguimiento" para niños de 2 a 6 años de edad. Con el fin de monitorear el territorio recorrido hacia le eliminación del sarampión, se ha hecho un esfuerzo por fortalecer la vigilancia de la enfermedad, de la cual forma parte la investigación de casos sospechados.

Tanto la campaña general de "puesta al día" como la de "seguimiento" alcanzaron coberturas de más de $98 \%$ en los grupos de edad a los que fueron dirigidas. El programa de vacunación de rutina también ha mantenido una alta cobertura. La alta inmunidad poblacional contra el sarampión lograda mediante estas estrategias de vacunación dio por resultado una rápida reducción de la incidencia de la enfermedad. De 1989 a 1992, se notificaron menos de 20 casos anuales confirmados por laboratorios. En Cuba, el último caso confirmado por pruebas serológicas se presentó en julio de 1993.

La estrategia para la eliminación del sarampión que se ha aplicado en Cuba ha interrumpido la transmisión de la enfermedad y ha impedido la circulación del virus causante en la isla. La experiencia de Cuba con la eliminación del sarampión sugiere que si se aplica una estrategia apropiada de vacunación, se puede erradicar el sarampión del mundo entero.

El virus del sarampión llegó a Cuba, importado por exploradores europeos, hace casi 500 años (1). Los registros que se han llevado en la isla desde principios del presente siglo revelan la pre-

1 Ministerio de Salud Pública, La Habana, Cuba.

2 Instituto Pedro Kourí, La Habana, Cuba.

3 Departamento de Salud del Condado de Los Ángeles, California.

4 Organización Panamericana de la Salud, Programa Especial de Vacunas e Inmunización, Washington, DC, EUA. sencia de brotes y epidemias de sarampión, acompañadas invariablemente de altas tasas de morbilidad y mortalidad. En 1980 se estableció que la eliminación del virus del sarampión era una de las prioridades de salud de Cuba. El presente trabajo tiene por objeto hacer un repaso de la epidemiología de la enfermedad en territorio cubano y describir la estrategia especial para la eliminación del sarampión que se creó y puso en marcha en el país.

\section{ANTECEDENTES HISTÓRICOS}

El sarampión sigue siendo una de las principales causas de morbilidad y mortalidad infantiles, especialmente en países en desarrollo. La OMS estima que, pese a la disponibilidad de una vacuna eficaz y barata, más de 1 millón de niños mueren de sarampión en el mundo cada año (2). Además, las secuelas que deja la enfermedad siguen afectando al individuo 
durante varios meses después de la infección (3).

El sarampión es una de las enfermedades víricas más contagiosas que afectan al ser humano (4). Antes de la creación de la vacuna, solía haber epidemias cada 2 ó 3 años en las zonas de más densidad poblacional y la infección se producía en todas partes y afectaba principalmente a lactantes y niños pequeños. El virus se transmite cuando el aerosol de las vías respiratorias de personas infectadas se deposita en las membranas mucosas o en el árbol respiratorio o conjuntivas de individuos susceptibles. El paciente se encuentra en su estado más contagioso durante la fase prodrómica de la enfermedad y antes de los primeros síntomas, que suelen ser la fiebre y la erupción cutánea. La transmisibilidad se reduce notablemente después de la erupción (5).

La vacuna a base de virus vivos atenuados, que fue autorizada por primera vez en los Estados Unidos de América (EUA) en 1963, se usaba ampliamente en muchos países a fines de los años setenta (6). Se han documentado casos en que la protección conferida por la vacuna ha durado 20 años o más, pero se cree que la inmunidad después de la vacunación puede perdurar toda la vida (7). La vacuna antisarampionosa tiene poca eficacia antes del noveno mes de vida debido a su neutralización por anticuerpos maternos tipo IgG adquiridos pasivamente por el recién nacido. La eficacia de la vacuna aumenta progresivamente después de esta edad y llega a un máximo entre los 9 y los 12 años. En general, la eficacia de la vacuna parece ser de $90 \%$ a $95 \%(8)$.

La amplia cobertura con la vacuna antisarampionosa en los programas rutinarios de vacunación ha redundado en marcadas reducciones de la incidencia del sarampión en muchos países, observándose en ocasiones una disminución de 99\% del número de casos notificados anualmente. Esta extraordinaria reducción de la circulación del virus, combinada con el éxito de las iniciativas de erradicación mundial de la viruela, estimuló el diálogo a principios de la década de los ochenta sobre la factibilidad de erradicar el sarampión del mundo entero $(9,10)$.

A pesar de los grandes avances en el mejoramiento del control del sarampión después de la creación de la vacuna, se siguieron produciendo brotes en países que habían logrado una alta cobertura en sus campañas de vacunación (11-14). Estas experiencias sugieren que la estrategia de administrar una sola dosis de vacuna en los servicios de salud ofrecidos rutinariamente puede resultar eficaz para reducir notablemente la circulación del virus del sarampión, pero no para eliminar la enfermedad. Resultaba obvio que hacía falta poner en marcha estrategias adicionales a fin de mejorar el control del sarampión y lograr eliminarlo. A la luz de estos datos, un repaso de las actividades adoptadas en Cuba para eliminar el sarampión puede servir como fuente de lecciones importantes para otros países que enfrentan problemas similares y están deseosos de adoptar una estrategia novedosa para controlar la enfermedad.

\section{EL SARAMPIÓN EN CUBA}

Antes de proponerse la meta de eliminar el sarampión, Cuba ya había tenido experiencia en el control y la eliminación de otras enfermedades prevenibles mediante vacunación (15). La viruela se eliminó de la isla en 1923 gracias a campañas de vacunación en masa. La eliminación de la poliomielitis del territorio cubano en 1962 se logró mediante campañas de vacunación en masa con la vacuna oral, junto con una intensa vigilancia de casos con parálisis flácida aguda $(16,17)$. A fin de monitorear los resultados de estos programas para la eliminación de enfermedades, se establecieron sistemas de vigilancia para investigar casos en que se sospecha una enfermedad prevenible por vacunación.

De 1933 hasta 1960 se notificó una baja incidencia de sarampión en Cuba, pese a la dudosa calidad de los datos de vigilancia y a que la incidencia de la enfermedad puede haberse subnoti- ficado enormemente. Durante los años sesenta el número de casos de sarampión notificados aumentó mucho en comparación con años anteriores, aunque puede que gran parte de este aumento se haya debido a una mejor vigilancia. Durante estos años el sarampión mostró fluctuaciones estacionales, con su mayor incidencia en los últimos meses de invierno o los primeros de la primavera. En los años sesenta, los niños menores de 5 años habían tenido las mayores tasas de incidencia de cualquier grupo de edad. En los años ochenta, los niños de 5 a 9 años estaban en mayor riesgo. Las tasas de letalidad se han mantenido bajas y han sido menores de $1 \%$ desde 1964.

La vacuna antisarampionosa se empezó a aplicar en Cuba en 1971 y la primera estrategia para el control del sarampión se puso en marcha entre 1971 y 1979. La población a la que se aplicó la vacuna por medio de los servicios de atención primaria fue la de los niños de 6 meses a 5 años de edad. A pesar de los adelantos alcanzados cada año en lo que respecta a la cobertura con la vacuna (en 1979 la cobertura de niños de 1 año de edad fue de $53 \%$ ), a fines de los años setenta seguía habiendo brotes de sarampión periódicamente, entre ellos uno que se produjo en 1977, en que se notificaron más de 25000 casos (figura 1).

En vista de que el virus del sarampión seguía circulando, se implantó una segunda estrategia para el control de la enfermedad durante el período de 1980 a 1985 . Esta estrategia consistía en vacunar a los niños que tuvieran de 9 meses a 14 años de edad. Se esperaba conseguir una cobertura de $90 \%$ mediante la provisión de la vacuna en los servicios de atención primaria. Aunque en promedio la cobertura con la vacuna antisarampionosa fue de $77,5 \%$ durante este período, la transmisión no se detuvo. En 1982 se notificaron más de 23000 casos de sarampión y en el período de 1983 a 1985 se notificaron 3000 casos anualmente.

A pesar de estas estrategias de control, la cobertura con la vacuna siguió siendo escasa y el virus del sarampión 
FIGURA 1. Casos de sarampión notificados por mes y cobertura con vacuna triple contra el sarampión, la parotiditis y la rubéola (SPR). Cuba, 1971-1997

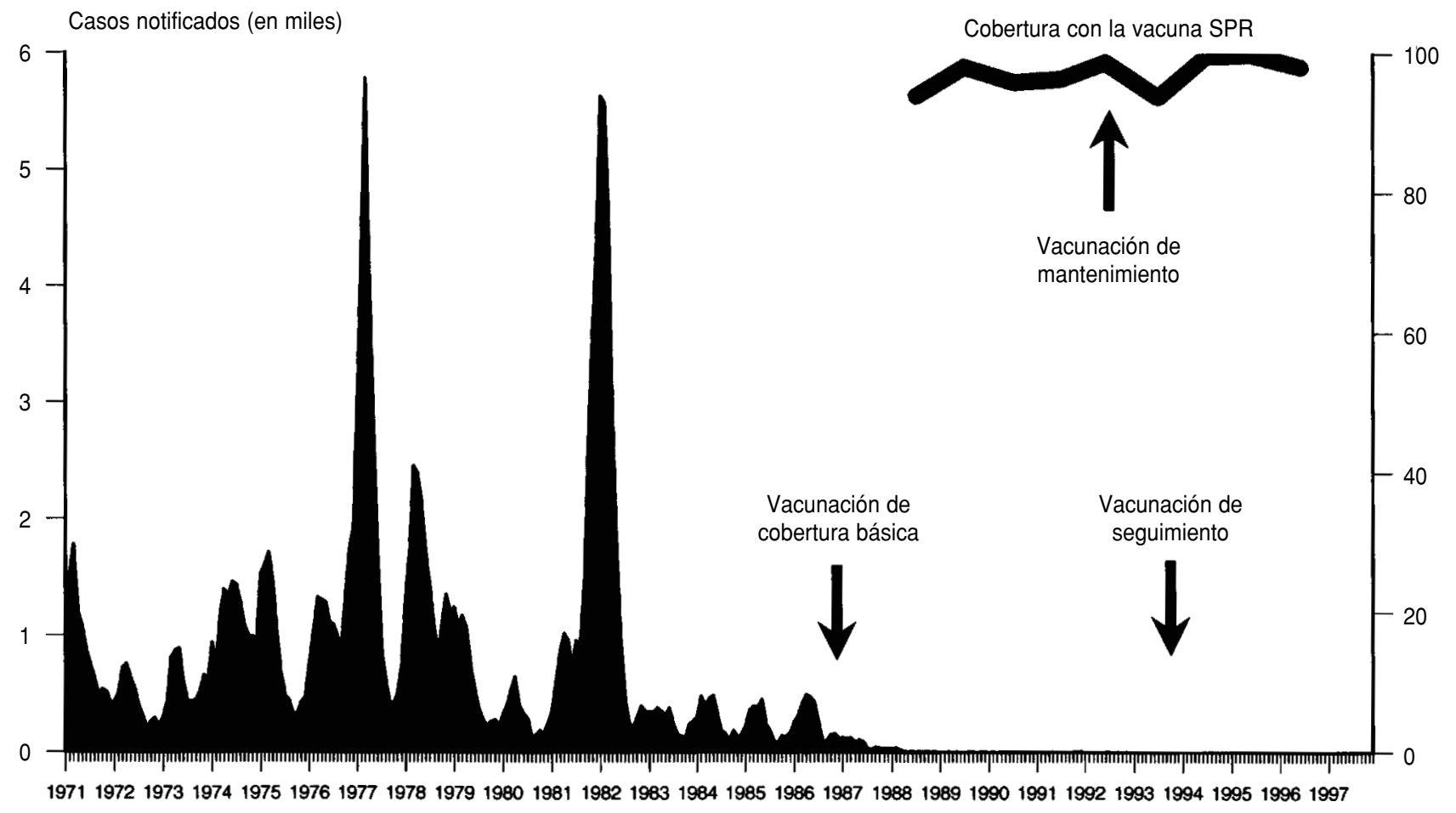

Mes y año

Fuente: Ministerio de Salud de Cuba.

continuó circulando entre los niños que no estaban comprendidos en los grupos de edad que debían vacunarse. Por añadidura, había pruebas de que los anticuerpos maternos interferían con las tasas de seroconversión sarampionosa en lactantes vacunados antes de cumplir un año. En el transcurso de esos años la vigilancia de casos de sarampión era pobre y rutinaria y se investigaban muy pocos de los casos notificados.

En 1985, un grupo de asesores de la OPS estudió la situación del sarampión en Cuba y propuso un enfoque novedoso para eliminar la enfermedad. Se trataba de una estrategia a base de efectuar periódicamente grandes campañas de vacunación antisarampionosa destinadas a la cobertura de niños de diferentes cohortes de edad, más la intensificación de las actividades de vigilancia. La estrategia serviría para complementar las prácticas de vacunación infantil ya en marcha en los servicios básicos de salud. El Ministerio de Salud de Cuba, apoyándose en las recomendaciones de la OPS, sus experiencias anteriores con las actividades de control del sarampión y la experiencia adquirida a partir de los programas para la eliminación de otras enfermedades que ya habían dado buenos resultados en Cuba, creó y puso en práctica una nueva estrategia para eliminar el sarampión.

\section{MATERIALES Y MÉTODOS}

\section{La vacunación contra el sarampión}

La estrategia cubana para la eliminación del sarampión tiene tres componentes esenciales. Primero se llevó a cabo una sola campaña de vacunación de alcance nacional destinada a "poner al día" la cobertura de todos los niños de 1 a 14 años, independientemente de sus antecedentes de vacunación o enfermedad, con el fin de aumentar rápidamente la inmunidad poblacional en varias cohortes de edad y reducir la cadena de transmisión. En segundo lugar se reforzaron los servicios básicos de vacunación y se procuró vacunar a un mínimo de $95 \%$ de los niños de un año de edad. El logro y mantenimiento de una alta cobertura en niños menores de un año por medio de los servicios básicos de salud han recibido el nombre de vacunación "de mantenimiento". Por último, se efectuarán campañas periódicas a gran escala, dirigidas a niños de 2 a 6 años de edad con o sin antecedentes de vacunación o enfermedad, a fin de evitar que el número de niños preescolares susceptibles llegue a niveles peligrosos capa- 
ces de aumentar el riesgo de un brote de sarampión.

No se fijó el intervalo entre las campañas "de seguimiento", pues se determinó que variaría según la cobertura con la vacuna de los menores de un año vacunados en los servicios de salud de uso habitual. En general, se llevarían a cabo estas campañas cuando el número de niños preescolares susceptibles que hubieran nacido después de la última campaña de "seguimiento" llegara a aproximarse al número promedio de niños nacidos en una cohorte anual.

\section{La vigilancia del sarampión}

Antes de 1959, la vigilancia del sarampión se hacía pasivamente, sin mayor esfuerzo por mejorar la notificación de casos. Después de ese año se aceleraron las medidas de vigilancia de todas las enfermedades de notificación obligatoria, incluidas las que pueden prevenirse por vacunación. Se enseñó a los médicos a reconocer diversas enfermedades y se elaboraron formularios de notificación. No obstante, faltaban lineamientos concretos sobre la manera de investigar casos con sospecha de sarampión y se disponía de pocos recursos de laboratorio para confirmar el agente etiológico.

En 1988 se establecieron y diseminaron en todos los niveles del sistema de salud lineamientos detallados para la vigilancia e investigación de casos. En lo fundamental, estos lineamientos hacían responsable a cada médico de cabecera por la notificación de todo paciente con fiebre y erupción cutánea a la clínica de salud pública local. El personal de salud, con participación de un epidemiólogo en ese nivel del sistema, debía ayudar al médico general a investigar los casos con sospecha de sarampión y la notificación debía hacerse a las autoridades del nivel provincial en un plazo máximo de 24 horas. Estas autoridades debían, a su vez, hacer la notificación a las autoridades nacionales en un máximo de 24 horas.

Se adoptaron definiciones estandarizadas para la vigilancia de casos de sarampión. Se determinó llamarle caso sospechado al de un paciente febril con erupción cutánea generalizada de tipo maculopapuloso, más tos, coriza, o conjuntivitis. Se denominó caso confirmado a un caso sospechado cuya infección sarampionosa se hubiera verificado mediante pruebas de laboratorio o que tuviera vinculación epidemiológica con otro caso confirmado de sarampión.

Para cada caso sospechado de sarampión se debían anotar los antecedentes epidemiológicos y clínicos y obtenerse muestras de suero durante las fases aguda y de convalecencia. Los pacientes debían permanecer en aislamiento domiciliario durante 5 días. Por añadidura, todos los contactos dentro del domicilio que tuvieran menos de 15 años de edad debían ser vacunados de inmediato si no se disponía de documentación en que constara que ya habían recibido la vacuna antisarampionosa.

En términos generales, los casos sospechados de sarampión y rubéola se manejaban como si se tratara de una misma afección clínica. Los sueros pareados obtenidos de casos con sospecha de sarampión o rubéola se sometían a pruebas de inhibición de la hemaglutinación (IH) para detectar la conversión serológica (títulos de IgG). En 1990 se empezaron a efectuar electroinmunoensayos enzimáticos (ELISA) para detectar IgG contra los virus del sarampión y la rubéola. Todas las muestras positivas a rubéola o sarampión al someterse al ELISA se volvieron a someter a pruebas de verificación, que fueron la $\mathrm{IH}$ y un ensayo de neutralización. En 1995 se introdujo el uso de un ELISA de captura para detectar IgM contra el virus del sarampión. Con el fin de aprovechar mejor los recursos limitados, desde 1994 la notificación de casos y su investigación en el laboratorio se han efectuado solamente en pacientes con fuerte sospecha clínica de sarampión o rubéola.

El país tenía 480 centros de vigilancia dispersos por todo el territorio nacional y por lo menos una unidad notificadora en cada uno de los 169 condados nacionales. Cada centro de notificación informaba semanalmente al Ministerio de Salud si había o no casos con sospecha de tener sarampión. Se elaboraron formularios de notificación con secciones para los antecedentes demográficos, clínicos y de vacunación. Estos formularios fueron llenados para cada paciente con sospecha de infección sarampionosa.

\section{RESULTADOS}

\section{Vacunación contra el sarampión}

De octubre de 1986 a marzo de 1987, Cuba llevó a cabo una campaña de "puesta al día" en la vacunación antisarampionosa y para ello utilizó la vacuna combinada contra el sarampión, la parotiditis y la rubéola (SPR). La campaña se dirigió a todos los niños de 1 a 14 años de edad, independientemente de sus antecedentes de vacunación o de infección sarampionosa. El personal de salud de los centros de atención y los médicos generales asignados a "áreas de circunscripción" (pequeñas zonas de captación de pacientes con aproximadamente 600 personas) fueron los responsables de llevar a cabo la campaña, que duró 6 meses. Un total de 2,5 millones de niños fueron la población objeto de la vacuna antisarampionosa. Durante la campaña, 2461329 niños fueron vacunados y se estima que la cobertura total fue de $97,6 \%$.

En noviembre de 1993 se puso en marcha una campaña "de seguimiento" dirigida a todos los niños de 2 a 6 años de edad, independientemente de sus antecedentes de vacunación o de enfermedad sarampionosa. Cerca de 900000 niños fueron la población objeto de una vacuna antisarampionosa a base de un solo antígeno. Durante la campaña, 883168 niños recibieron esta vacuna, lo cual equivalió a una cobertura de $98 \%$. Cada una de las 15 provincias del territorio cubano alcanzó una cobertura mayor de 95\%.

Desde 1986 se ha procurado mejorar la cobertura con la vacuna administrada rutinariamente pidiendo a cada médico general que lleve un registro 
de la inmunización de todos los niños menores de 15 años que vivan en la zona de su jurisdicción. La cobertura con la vacuna MMR administrada habitualmente en los servicios de salud alcanzó niveles muy altos en niños de un año de edad, y entre 1988 y 1996 se logró en Cuba una cobertura promedio de $98 \%$ (figura 1 ).

\section{Vigilancia de casos de sarampión}

A partir de 1988 se empezó a investigar cuidadosamente a la mayoría de los casos con sospecha de sarampión o rubéola, y ello incluía la extracción de las debidas muestras de sangre para realizar las pruebas de laboratorio. La notificación de pacientes con sospecha de infección sarampionosa o rubéola aumentó de 122 en 1988 a una cifra máxima de 1319 en 1991 (cuadro 1).

Una vez terminada la campaña de "puesta al día", los casos confirmados de sarampión se redujeron en 1988 a 122 , cifra que fue la más baja en 20 años. Desde 1989, y a pesar de las mejoras logradas en la vigilancia, menos de 20 casos confirmados por pruebas serológicas se han notificado cada año. En 1992 hubo un total de 12 casos de sarampión confirmados por pruebas de laboratorio. En 1993 hubo dos casos confirmados por laboratorio, en ninguno de los cuales se pudo determinar la fuente de infección, pero no transmitieron la infección a casos nuevos. Cuba notificó el último caso de sarampión confirmado mediante pruebas de laboratorio en julio de 1993. También ha habido en Cuba una marcada reducción de los casos de rubéola confirmados en el laboratorio (cuadro 1).

De 1989 a 1992, un total de 63 casos confirmados tenían información sobre sus vacunaciones previas. De ellos, 29 (47\%) habían recibido la vacuna antisarampionosa. No se han presentado defunciones por sarampión en Cuba desde 1985. Para los años de 1988 a 1990 , no se pudo identificar la fuente de ninguno de los 36 casos de sarampión confirmados mediante pruebas de laboratorio. En 1991, cinco de los 22 casos confirmados estaban vinculados en sentido temporal y pertenecieron a la provincia de Güira. De los 17 casos restantes, seis se produjeron en la provincia de Santiago, pero no se pudo determinar la fuente de la infección.
En 1992, dos de los 12 casos confirmados en el laboratorio estaban vinculados. Los restantes, incluidos cinco casos notificados en la provincia de Santiago, carecieron de una fuente identificable.

\section{DISCUSIÓN}

Pese la esmerada vigilancia de casos de sarampión, ya hace más de 4 años que en Cuba no se notifica ningún caso de sarampión confirmado en el laboratorio. La estrategia para la eliminación del sarampión que Cuba puso en marcha, la cual ha consistido en alcanzar con rapidez y mantener elevada la inmunidad poblacional contra el sarampión mediante una combinación de servicios rutinarios de vacunación y extensas campañas de vacunación efectuadas periódicamente, parece haber interrumpido la circulación del virus del sarampión en la isla. Por añadidura, esta interrupción de la transmisión se ha mantenido por varios años.

Anteriormente se han descrito resultados similares a los de Cuba, a raíz de campañas de vacunación a gran escala

CUADRO 1. Investigación de pacientes con sospecha de tener sarampión y rubéola, utilizando pruebas de laboratorio. Cuba, 1988-1997a

\begin{tabular}{|c|c|c|c|c|c|c|c|c|c|}
\hline \multirow[b]{2}{*}{ Año } & \multirow{2}{*}{$\begin{array}{c}\text { Pacientes } \\
\text { con sospecha de } \\
\text { sarampión, rubéola, } \\
\text { o ambas enfermedades }\end{array}$} & \multicolumn{2}{|c|}{$\begin{array}{c}\text { Pacientes } \\
\text { de quienes se } \\
\text { obtuvieron muestras } \\
\text { de suero adecuadas }\end{array}$} & \multicolumn{3}{|c|}{ Seropositividad al sarampión } & \multicolumn{3}{|c|}{ Seropositividad a la rubéola } \\
\hline & & No. & $\%$ & $n$ & No. & $\%$ & $n$ & No. & $\%$ \\
\hline 1988 & 122 & 163 & 76,5 & 50 & 8 & 16,0 & 163 & 27 & 16,6 \\
\hline 1989 & 211 & 176 & 83,4 & 176 & 12 & 6,8 & 175 & 165 & 94,3 \\
\hline 1990 & 668 & 630 & 94,3 & 630 & 17 & 2,7 & 630 & 25 & 4,0 \\
\hline 1991 & 1319 & 1215 & 92,1 & 1215 & 22 & 1,8 & 1215 & 25 & 2,1 \\
\hline 1992 & 1104 & 1007 & 91,2 & 1007 & 12 & 1,2 & 1007 & 4 & 0,4 \\
\hline 1993 & 633 & 595 & 94,0 & 595 & 2 & 0,3 & 595 & 8 & 1,3 \\
\hline 1994 & 359 & 324 & 90,3 & 324 & 0 & 0 & 324 & 4 & 1,2 \\
\hline 1995 & 162 & 146 & 90,1 & 146 & 0 & 0 & 146 & 3 & 2,1 \\
\hline 1996 & 146 & 132 & 90,4 & 132 & 0 & 0 & 132 & 0 & 0 \\
\hline 1997 & 348 & 327 & 94,0 & 327 & 0 & 0 & 327 & 0 & 0 \\
\hline
\end{tabular}

a Los datos son de a partir del 25 de febrero de 1988.

Nota: De 1988 a 1993, todos los pacientes con fiebre y erupción cutánea, salvo aquellos cuyos síntomas sugerían varicela o escarlatina, fueron estudiados mediante pruebas de laboratorio. A partir de 1994, solamente el suero de pacientes con sospecha de rubéola o sarampión se sometió a estas pruebas.

De 1988 a 1990, las muestras se sometieron a la prueba de inhibición de la hemaglutinación para la detección de rubéola y sarampión. De 1991 a 1995 , las muestras se estudiaron mediante ELISA detectores de anticuerpos contra los virus del sarampión y la rubéola. Los sueros que dieron resultados positivos en esta prueba se sometieron, con fines de verificación, a la prueba de inhibición de la hemaglutinación, a la prueba de neutralización y a ELISA de captura. 
para eliminar el sarampión, en Gambia en las postrimerías de los años sesenta y más recientemente en otros países de América Latina y el Caribe $(18,19)$. Gambia se mantuvo libre de casos de sarampión durante un total de 3 años. No obstante, a principios de los años setenta la iniciativa para la eliminación del sarampión se suspendió por falta de fondos, y a raíz de la entrada del virus de un país vecino, el sarampión se convirtió nuevamente en una enfermedad endémica en ese país (20).

Algunos modelos matemáticos han ilustrado las ventajas de adoptar campañas de vacunación a gran escala para aumentar rápidamente la inmunidad poblacional contra el sarampión e interrumpir la transmisión del virus que lo causa (21). Se estima que se necesita una inmunidad poblacional mínima de 93\% para interrumpir o prevenir la circulación del virus del sarampión (22). Si la inmunidad poblacional asciende a un porcentaje superior, es muy baja la probabilidad de que un individuo con sarampión entre en contacto con una persona susceptible a la enfermedad, y por lo tanto debería cesar la circulación del virus causal (23). La campaña de "puesta al día" permite lograr una rápido aumento de la inmunidad poblacional en varias cohortes de edad. Si por ese medio se logra una cobertura lo suficientemente alta, puede esperarse que la circulación del virus del sarampión que ya circula quede interrumpida.

La vacunación rutinaria de los niños cuando cumplen un año de edad y las campañas "de seguimiento" efectuadas periódicamente son medidas que garantizan el mantenimiento de un alto nivel de inmunidad poblacional a lo largo del tiempo en la población preescolar. Si el virus del sarampión llegara a reintroducirse en la población por importación desde una zona endémica, la probabilidad de que se restablezca la circulación del virus seguirá siendo remota.

En Cuba ha habido cambios importantes en las metodología aplicada para la vigilancia del sarampión, y es necesario tenerlos en cuenta a la hora de interpretar los datos obtenidos. Antes de 1987 la vigilancia de los casos de sarampión solía ser pasiva y no se confirmaban los casos mediante pruebas de laboratorio. Es muy probable que se haya producido una subnotificación de casos y que algunos de los casos notificados no hayan sido verdaderamente de sarampión. Por consiguiente, los casos notificados pueden haber llevado a un cálculo demasiado bajo o demasiado alto de la verdadera incidencia de la enfermedad.

Desde 1987, sin embargo, se ha procurado mejorar la sensibilidad y especificidad del sistema de notificación de casos de sarampión mediante una vigilancia activa y la investigación de todos los casos notificados.

Pese a no haberse examinado detenidamente en el presente trabajo, el uso de la vacuna triple (SPR) durante la campaña inicial cuyo objeto fue la "puesta al día" y en los servicios habituales de vacunación ha dado por resultado grandes reducciones del número de casos de rubéola y parotiditis confirmados por los laboratorios. A fin de prevenir el síndrome congénito por rubéola (SCR), el Ministerio de Salud de Cuba también ha lanzado iniciativas destinadas a lograr niveles altos de inmunidad contra la rubéola en mujeres en edad reproductiva. La experiencia de Cuba en la eliminación de la rubéola y del SCR se examinarán en un trabajo posterior.

Además de su estrategia de vacunación tan eficaz, otros factores han ayudado a Cuba a eliminar el sarampión como problema de salud pública. El tamaño reducido del país y la buena organización de su sistema de atención primaria le han permitido alcanzar y mantener una cobertura por vacunación más alta que la que se podría haber obtenido en otras áreas. Por tratarse de una isla, también es de esperar que sea menor la importación del virus del sarampión desde zonas endémicas. En años recientes Cuba ha tenido muy poca inmigración de personas procedentes de lugares donde el sarampión es endémico. Muchos de los casos confirmados por pruebas de laboratorio que se han presentado después de las extensas campañas de vacunación fueron casos aislados en un sentido temporal y territorial. Se desconoce la fuente de infección en estos casos y ninguno ha sido responsable de la transmisión del virus. Es posible que se hayan producido importaciones no detectadas de otros países, que no se hayan identificado debidamente casos nuevos de sarampión, o que se hayan obtenido resultados de laboratorio negativos falsos.

El virus del sarampión sigue circulando en muchas partes del mundo y es muy probable que algunos de los casos confirmados de la enfermedad se deban a virus importados de otros países. Dado el estado avanzado del sistema de atención primaria en Cuba, no es muy probable que estos casos confirmados aislados sean casos que no fueron detectados durante un brote de sarampión más extenso. Merece atención la posibilidad de que estos casos hayan tenido resultados serológicos negativos falsos. De hecho, el valor predictivo positivo de una prueba para el diagnóstico del sarampión es bajo, por definición, cuando no circula el virus causal.

Sumada a los resultados teóricos obtenidos con modelos matemáticos y a la experiencia de campo de otros países del Caribe y de América Latina, la experiencia de Cuba ofrece pruebas epidemiológicas convincentes de la factibilidad de interrumpir la circulación del virus del sarampión en el corto plazo y de mantener la interrupción a lo largo del tiempo si se pone en marcha una estrategia adecuada de vacunación con la vacuna antisarampionosa estándar disponible. Actualmente otras partes del mundo enfrentan el reto de aprender de la experiencia que han tenido Cuba y otros países latinoamericanos con la eliminación del sarampión, adaptando la estrategia de vacunación a sus propias circunstancias con el fin de encaminarse hacia la meta de eliminar esa enfermedad del mundo entero. 
1. Bianchine PJ, Russo TA. The role of epidemic infectious diseases in the discovery of America. Allergy Proc 1992;13(5):225-232.

2. World Health Organization. Expanded Programme on Immunization: Accelerated measles strategies. Wkly Epidemiol Rec 1994;69 (31):229-234.

3. Smith EA, Foster SO. Measles in areas of malnutrition. J Nigeria Med Assoc 1970;7:16-18.

4. Hope-Simpson RE. Infectiousness of communicable diseases in the household. Lancet 1952;2:549-554.

5. Krugman S, Katz SL, Gershon AA, Wilfert C. Infectious diseases of children. Octava edición. St. Louis: C. V. Mosby; 1985.

6. Krugman S, Giles JP, Jacobs AM, Friedman $H$. Studies with a further attenuated live measles-virus vaccine. Pediatrics 1963;31: 919-928

7. Markowitz LE, Preblud SR, Fine PE, Orenstein WA. Duration of measles vaccineinduced immunity. Pediatr Infect Dis J 1990; 9(2):101-110

8. Bellini WJ, Rota JS, Rota PA. Virology of measles virus. J Infect Dis 1994;170(suppl 1): S15-S23.

9. Hopkins DR, Hinman AR, Koplan JP, Lane JM. The case for global measles eradication. Lancet 1982;1(8286):1396-1398.

10. Foege $\mathrm{WH}$. The global elimination of measles. Public Health Rep 1982;97(5):402-405.

11. Markowitz LE, Preblud SR, Orenstein WA, Rovira EZ, Adams NC, Hawkins CE, et al.
Patterns of transmission in measles outbreaks in the United States, 1985-1986. N Engl J Med 1989;320(2):75-81.

12. Hersh BS, Markowitz LE, Maes EF, Funkhouser AW, Baushman AL, Sirotkin BI, et al. The geographic distribution of measles in the United States, 1980-1989. JAMA 1992;267: 1936-1941.

13. Clements CJ, Strassburg M, Cutts FT, Torel C. The epidemiology of measles. World Health Stat Q 1992;45(2-3):285-291.

14. Chen RT, Weierbach R, Bisoffi Z, Cutts F, Rhodes $\mathrm{P}$, Ramaroson $\mathrm{S}$, et al. A posthoneymoon period measles outbreak in Muyinga province, Burundi. Int J Epidemiol 1994;23(1):185-193.

15. Molinert HT, Rodríguez R, Galindo M. Principales aspectos del programa nacional de inmunización de la República de Cuba. La Habana: Ministerio de Salud; 1992.

16. Cruz RR. Cuba: Mass polio vaccination program 1962-1982. Rev Infect Dis 1984;6(suppl 2):S408-S412.

17. Lago PM, Bravo JR, Andrus JK, Comellas $\mathrm{MM}$, Galindo MA, de Quadros CA, et al. Lessons from Cuba: Mass campaign administration of trivalent oral poliovirus vaccine and seroprevalence of poliovirus neutralizing antibodies. Bull World Health Organ 1994; 72(2):221-225

18. Foege WH. Measles vaccination in Africa. En: Proceedings of the International Conference on the Application of Vaccines Against Viral, Rickettsial, and Bacterial Diseases of Man. Washington, DC: Pan American Health Organization; 1971. pp. 207-212. (Scientific Publication 226).

19. de Quadros CA, Olivé JM, Hersh BS, Strassburg MA, Henderson DA, Brandling-Bennett $\mathrm{D}$, et al. Measles elimination in the Americas: Evolving strategies. JAMA 1996;275(3): 224-229.

20. Williams PJ, Hull HF. Status of measles in Gambia, 1981. Rev Infect Dis 1983;5(3): 391-394.

21. Agur Z, Was Cojocaru L, Mazor G, Anderson RM, Danon YL. Pulse mass measles vaccination across age cohorts. Proc Natl Acad Sci 1993;90(24):11698-11702.

22. Nokes DJ, Williams JR, Butler AR. Towards eradication of measles virus: Global progress and strategy evaluation. Veterinary Microbiol 1994;44(2-4):333-350.

23. Fine PE. Herd immunity: History, theory, practice. Epidemiol Rev 1993;15(2):265-302.

Manuscrito recibido el 7 de mayo de 1997 y aceptado para publicación en versión revisada el 23 de abril de 1998.

ABSTRACT The vaccine against measles came into use in Cuba in 1971. During the seventies, a new early strategy for measles control was established, and it was followed by further efforts in the early eighties. Despite improvements to the control program, disease outbreaks continued to occur. In 1986, after examining the experience acquired through the control initiatives that were already in place, a new measles vaccination strategy was adopted.

In time, the new vaccination strategy against measles came to have three main components: first, a single vaccination "catching-up" campaign targeting children 1 to 14 years of age. Second, efforts were made to achieve and maintain high vaccine coverage through mandatory vaccination services for 12-month-old children ("maintenance vaccination"). Finally, periodic "follow-up" campaigns were carried out for children 2 to 6 years of age. Steps were taken, for the purpose of monitoring the progress made so far toward eliminating measles, to strengthen disease surveillance systems, including the screening of suspected cases.

The "catching-up" and "follow-up" campaigns both achieved greater than $98 \%$ coverage within targeted age groups. The routine vaccination program has also maintained high coverage. The high population immunity against measles that has been attained through these vaccination strategies has resulted in a rapid decrease in the incidence of the disease. From 1989 to 1992, less than 20 laboratory-confirmed cases were reported annually. In Cuba, the last case confirmed through serologic screening was reported in July 1993.

Cuba's strategy for measles elimination has interrupted disease transmission and kept the causal virus from circulating on the island. Cuba's experience with measles elimination suggests that if an appropriate vaccination strategy is applied, measles can be globally eradicated. 